PREPARED FOR THE U.S. DEPARTMENT OF ENERGY, UNDER CONTRACT DE-AC02-76CH03073

PPPL-3921

PPPL-3921

UC-70

A Triangular Finite Element with First-derivative Continuity Applied to Fusion MHD Applications

by

S.C. Jardin

January 2004

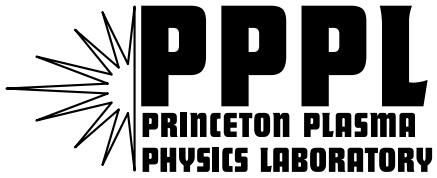

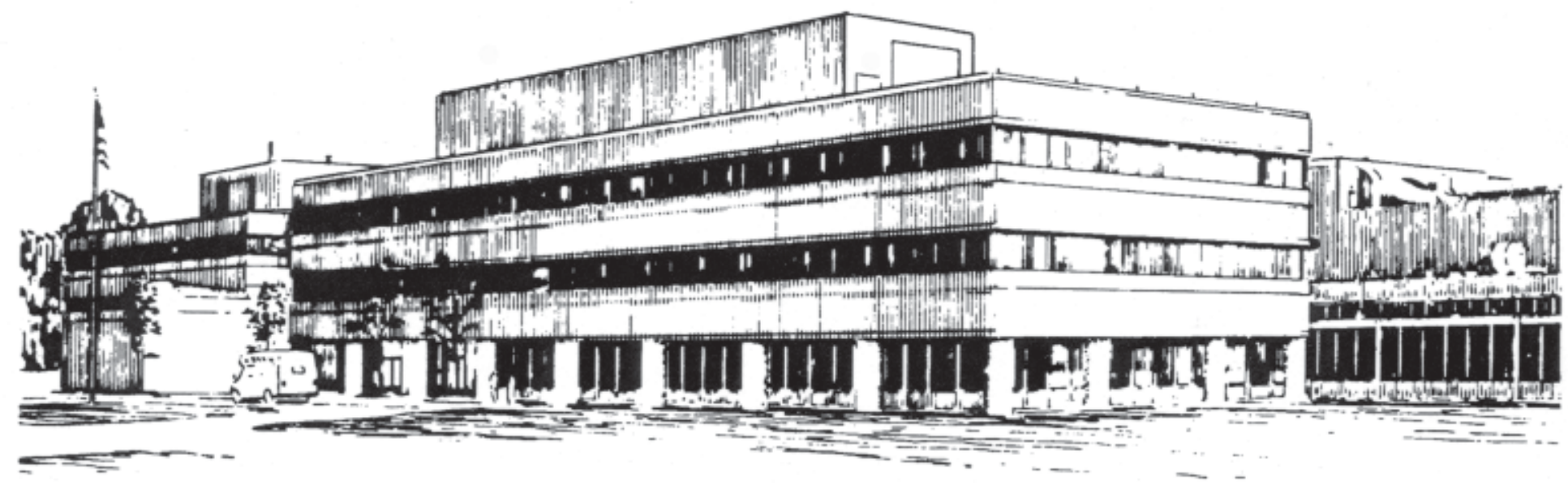

PRINCETON PLASMA PHYSICS LABORATORY PRINCETON UNIVERSITY, PRINCETON, NEW JERSEY 


\section{PPPL Reports Disclaimer}

This report was prepared as an account of work sponsored by an agency of the United States Government. Neither the United States Government nor any agency thereof, nor any of their employees, makes any warranty, express or implied, or assumes any legal liability or responsibility for the accuracy, completeness, or usefulness of any information, apparatus, product, or process disclosed, or represents that its use would not infringe privately owned rights. Reference herein to any specific commercial product, process, or service by trade name, trademark, manufacturer, or otherwise, does not necessarily constitute or imply its endorsement, recommendation, or favoring by the United States Government or any agency thereof. The views and opinions of authors expressed herein do not necessarily state or reflect those of the United States Government or any agency thereof.

\section{Availability}

This report is posted on the U.S. Department of Energy's Princeton Plasma Physics Laboratory Publications and Reports web site in Fiscal Year 2004. The home page for PPPL Reports and Publications is: http://www.pppl.gov/pub_report/

DOE and DOE Contractors can obtain copies of this report from:

U.S. Department of Energy

Office of Scientific and Technical Information

DOE Technical Information Services (DTIS)

P.O. Box 62

Oak Ridge, TN 37831

Telephone: (865) 576-8401

Fax: (865) 576-5728

Email: reports@adonis.osti.gov

This report is available to the general public from:

National Technical Information Service

U.S. Department of Commerce

5285 Port Royal Road

Springfield, VA 22161

Telephone: $1-800-553-6847$ or

(703) $605-6000$

Fax: (703) 321-8547

Internet: http://www.ntis.gov/ordering.htm 


\title{
A Triangular Finite Element with First-Derivative Continuity Applied to Fusion MHD Applications
}

\author{
S. C. Jardin \\ Princeton University \\ Plasma Physics Laboratory \\ P. O. Box 451 \\ Princeton, NJ 08543 \\ E-mail: jardin@princeton.edu
}

\begin{abstract}
We describe properties of the reduced quintic triangular finite element. The expansion used in the element will represent a complete quartic polynomial in two dimensions, and thus the error will be of order $h^{5}$ if the solution is sufficiently smooth. The quintic terms are constrained to enforce $C^{1}$ continuity across element boundaries, allowing their use with partial differential equations involving derivatives up to fourth order. There are only three unknowns per node in the global problem, which leads to lower rank matrices when compared with other high-order methods with similar accuracy but lower order continuity. The integrations to form the matrix elements are all done in closed form, even for the nonlinear terms. The element is shown to be well suited for elliptic problems, anisotropic diffusion, the Grad-Shafranov-Schlüter equation, and the timedependent MHD or extended MHD equations. The element is also well suited for 3D calculations when the third (angular) dimension is represented as a Fourier series.
\end{abstract}

\section{Keywords:}

Finite Elements, magnetohydrodynamics,

\section{Subject Classification:}

65P25, 77A05 


\section{Introduction}

Two dimensional (2D) finite elements are being used in a wide variety of fusion applications. Even in fully 3D calculations in toroidal geometry, it is common to use 2D elements in the poloidal plane, and to use either finite differences or a Fourier spectral representation in the toroidal angle.

While early work used primarily linear elements[1,2], it is now recognized that higher order elements offer significant advantages, and are essential to adequately represent highly anisotropic heat transport and other anisotropic processes[3,4,6]. However, the application of high-order elements to fusion problems has so far been restricted to the class of elements known as $C^{0}$ elements, which includes both the spectral and the Lagrange basis. These are constructed so as to have the unknown function continuous between elements, but none of its derivatives are forced to be continuous. The rational for this is that it is less complex to construct such elements and if the minimizing solution has high-order continuity, this solution will emerge from the Galerkin process without having to be specifically imposed.

However, there are clearly some advantages in using elements with higher order intrinsic continuity. We can expect that for a problem whose solution has continuous firstderivatives everywhere, i.e. satisfies $C^{1}$ continuity, fewer basis functions will be required

per element to approximate the true solution if the $C^{1}$ constraint is imposed in the construction of the basis functions, i.e. if the degrees of freedom that are not compatible with global $C^{1}$ continuity have been discarded from the outset. We can further expect that this will lead to smaller matrix sizes with similar sparseness patterns to the matrices that arise with the $C^{0}$ elements, and thus a more efficient solution procedure should result.

Also, many problems in extended MHD involve operators higher than second order in space. Examples of these are the viscosity operator in the vorticity equation, and the hyper-resistivity operator in the magnetic flux equation. The $C^{1}$ elements allow the treatment of fourth order operators by using the standard Galerkin technique of shifting two of the derivatives to the trial function, whereas this is not possible with the $C^{0}$ elements, which need to introduce auxiliary variables and expand them in finite elements. Thus, the $C^{1}$ elements can expect to have an additional efficiency and resultant smaller matrices since they do not need to introduce these auxiliary variables when third or fourth order derivatives are present. Conversely, in some cases several low order equations can be combined to produce a smaller number of higher order equations that can be approximated directly with these elements that possess higher order continuity.

We consider only triangular finite elements in this paper, in fact, only a particular triangular finite element known as the reduced quintic [6] (also called the Bell triangle $[7,8]$ and the TUBA 3 element [9]). This reflects a bias that triangular elements are more flexible for representing complex geometry, and can be easily refined as needed simply by dividing one triangle into three or more. It is especially efficient and convenient when the different triangles connect only via the vertices, and that is where all the unknowns are defined. With these constraints, and that of $C^{1}$ continuity, the reduced quintic 
element emerges. While this element has been used in structural engineering studies since the late 1960s [10], it has apparently been overlooked by the extended MHD community. Here we show that it has some real advantages, and should be seriously considered as a basis for contemporary computational models of extended MHD in magnetized plasmas.

\section{The Reduced Quintic Finite Element}

\section{A. The Elements}

Consider the reduced quintic 2D triangular finite element in the $x-y$ plane as depicted in Fig. 1. In each triangular element, the unknown function $\phi(x, y)$ is written as a general polynomial of $5^{\text {th }}$ degree in the local Cartesian coordinates $\xi$ and $\eta: \phi(\xi, \eta)=\sum_{i=1}^{20} a_{i} \xi^{m_{i}} \eta^{n_{i}}$ (where the exponents $m_{i}, n_{i}$ are given in Table 1) which would have 21 coefficients were there not additional constraints. Eighteen of the coefficients are determined from specifying the values $\phi, \phi_{x}, \phi_{y}, \phi_{x x}, \phi_{x y} ; \phi_{y y}$ at each of the 3 vertices, thus guaranteeing that globally all first and second derivatives will be continuous at each vertex. Since the onedimensional quintic polynomial along each edge is completely determined by these values specified at the endpoints, it is guaranteed that the expansion is continuous between elements.

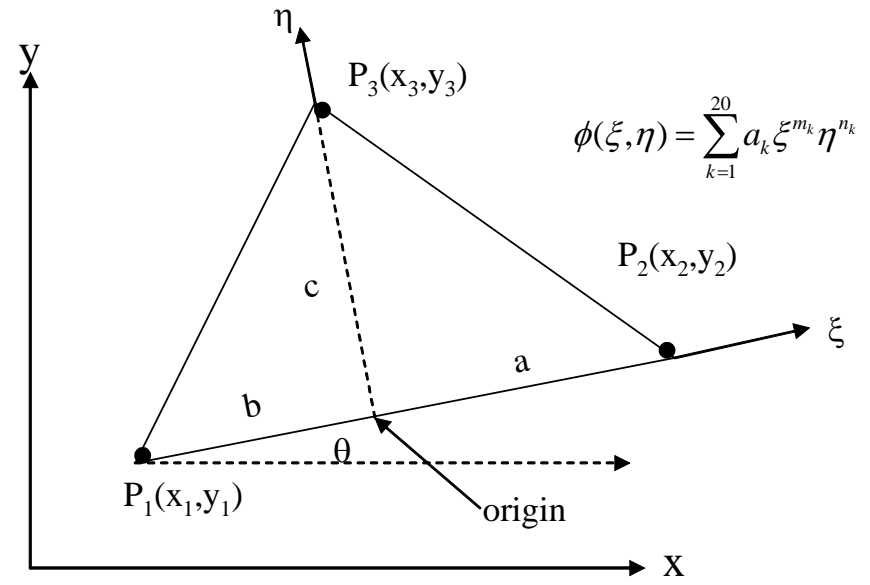

Figure 1: Reduced quintic finite element is defined by the 4 geometric parameters a, b, $\mathbf{c}, \theta$. A local $(\xi, \eta)$ Cartesian system is used. The function and first 2 derivatives are constrained at the 3 points, and $C^{1}$ continuity is imposed at the edges . Exponents $m_{i}$ and $n_{i}$ are given in table 1 .
The remaining three constraints come from the requirement that the normal derivative of $\phi$ at each edge, $\phi_{n}$, reduce to a onedimensional cubic polynomial along that edge. This implies that the two sets of nodal values completely determine $\phi_{n}$ everywhere on each edge, guaranteeing its continuity from one triangle to the next so that the element is $C^{1}$. One of these three constrains is trivial and has been used to reduce the number of terms from 21 to

20 in the sum.

Note that in imposing these continuity constraints, the expansion is no longer a complete quintic, but it does contain a complete quartic with additional constrained quintic coefficients to enforce $C^{1}$ continuity between elements. Thus the name, "reduced quintic". If the characteristic size of the element is $h$, then it follows from a local Taylor's 
series analysis that the approximation error in the unknown function, $\phi-\phi^{h}$, will be of order $h^{5}$, which leads to global $O\left(h^{5}\right)$ accuracy after integrating over the element.

\begin{tabular}{|l|l|l|l|l|l|l|l|l|l|l|l|}
\hline $\mathrm{k}$ & $\mathrm{m}_{\mathrm{k}}$ & $\mathrm{n}_{\mathrm{k}}$ & $\mathrm{k}$ & $\mathrm{m}_{\mathrm{k}}$ & $\mathrm{n}_{\mathrm{k}}$ & $\mathrm{k}$ & $\mathrm{m}_{\mathrm{k}}$ & $\mathrm{n}_{\mathrm{k}}$ & $\mathrm{k}$ & $\mathrm{m}_{\mathrm{k}}$ & $\mathrm{n}_{\mathrm{k}}$ \\
\hline 1 & 0 & 0 & 6 & 0 & 2 & 11 & 4 & 0 & 16 & 5 & 0 \\
\hline 2 & 1 & 0 & 7 & 3 & 0 & 12 & 3 & 1 & 17 & 3 & 2 \\
\hline 3 & 0 & 1 & 8 & 2 & 1 & 13 & 2 & 2 & 18 & 2 & 3 \\
\hline 4 & 2 & 0 & 9 & 1 & 2 & 14 & 1 & 3 & 19 & 1 & 4 \\
\hline 5 & 1 & 1 & 10 & 0 & 3 & 15 & 0 & 4 & 20 & 0 & 5 \\
\hline
\end{tabular}

Table 1: Exponents of $\xi$ and $\eta$ for the reduced quintic expansion $\phi(\xi, \eta)=\sum_{i=1}^{20} a_{i} \xi^{m_{i}} \eta^{n_{i}}$

Suppose that we are approximating a square domain by partitioning it into $n^{2}$ squares or $2 n^{2}$ triangles. The reduced quintic will asymptotically have $N=6 n^{2}$ unknowns, or three unknowns for each triangle. This scaling can be verified by the fact that if we introduce a new point into any triangle and connect it to the 3 nearby points; we will have generated 2 new triangles and introduced 6 new unknowns. We contrast this with linear elements that require only $\phi$ at the nodes and thus have $C^{0}$ continuity, $1 / 2$ unknown per triangle, and an approximation error of order $h^{2}$.

Another popular class of higher order 2D finite elements, that has only $C^{0}$ continuity enforced, is the Lagrangian elements. These use as a basis within each element a set of basis functions that are unity at a particular node and that vanish at all other nodes. Continuity requires that there be $M+1$ nodes along each side for an $M^{\text {th }}$ order polynomial element, with the remaining nodes being interior nodes (or "bubble nodes"). Thus for the Lagrangian elements, a quadratic element (6 coefficients per triangle) will have 3 nodes along each edge ( 2 vertex nodes and 1 edge node), a cubic element (10 coefficients per triangle) will have 4 nodes along each edge ( 2 vertex nodes and 2 edge nodes) and 1 interior node and a quartic element (15 coefficients per triangle) will have a total of 3 vertex nodes, 9 edge nodes, and 3 interior nodes. It is easily seen that these higher-order elements will asymptotically have 2, 41/2, and 8 unknowns per triangle (UK/T), respectively, (or 2, 31/2 and 5 if you discount the interior nodes that can be efficiently eliminated by static condensation). We summarize these $2 \mathrm{D}$ triangular elements in Table 2 .

\begin{tabular}{|l|l|l|l|l|l|l|l|}
\hline & $\begin{array}{l}\text { Vertex } \\
\text { nodes }\end{array}$ & $\begin{array}{l}\text { Line } \\
\text { nodes }\end{array}$ & $\begin{array}{l}\text { Interior } \\
\text { nodes }\end{array}$ & $\begin{array}{l}\text { accuracy } \\
\text { order } \mathrm{h}^{\mathrm{p}}\end{array}$ & $\mathrm{UK} / \mathrm{T}$ & $\mathrm{UK} / \mathrm{T}^{\#}$ & continuity \\
\hline linear element & 3 & 0 & 0 & 2 & $1 / 2$ & $1 / 2$ & $\mathrm{C}^{0}$ \\
\hline Lagrange quadratic & 3 & 3 & 0 & 3 & 2 & 2 & $\mathrm{C}^{0}$ \\
\hline Lagrange cubic & 3 & 6 & 1 & 4 & $41 / 2$ & $31 / 2$ & $\mathrm{C}^{0}$ \\
\hline Lagrange quartic & 3 & 9 & 3 & 5 & 8 & 5 & $\mathrm{C}^{0}$ \\
\hline reduced quintic & 18 & 0 & 0 & 5 & 3 & 3 & $\mathrm{C}^{1^{*}}$ \\
\hline
\end{tabular}

Table 2: Summary of properties of the reduced quintic and the low-order Lagrange elements. UK/T is the number of unknowns per triangle. * note $C^{2}$ continuity at nodes. $U K / T^{\#}$ is the number of unknowns per triangle, not counting interior nodes 


\section{B. Computations:}

It is shown in Appendix A that if we locally number the unknowns $\phi, \phi_{x}, \phi_{y}, \phi_{x x}, \phi_{x y}, \phi_{y y}$ at $P_{1}$ as $\Phi_{1}-\Phi_{6}$, at $P_{2}$ as $\Phi_{7}-\Phi_{12}$, and at $P_{3}$ as $\Phi_{13}-\Phi_{18}$, then the coefficients $a_{i}$ for a given element are determined uniquely by the relation:

$$
a_{i}=\sum_{j=1}^{18} g_{i, j} \Phi_{j}
$$

where the $20 \times 18$ matrix $g_{i, j}$ depends only on the shape and orientation of the individual triangle. Thus, the general expression for the unknown function $\phi$ in a given triangle is:

$$
\phi(\xi, \eta)=\sum_{i=1}^{20} a_{i} \xi^{m_{i}} \eta^{n_{i}}=\sum_{i=1}^{20} \sum_{j=1}^{18} g_{i, j} \Phi_{j} \xi^{m_{i}} \eta^{n_{i}}
$$

or,

$$
\phi(\xi, \eta)=\sum_{j=1}^{18} v_{j} \Phi_{j}
$$

We have defined the basis functions as

$$
v_{j} \equiv \sum_{i=1}^{20} g_{i, j} \xi^{m_{i}} \eta^{n_{i}}
$$

for $j=1,18$. The 18 basis functions for each triangle, as defined in Eq. (1.4) have the property that they have a unit value for either the function or one of it's first or second derivatives at one vertex and zero for the other quantities at this and the other nodes. They also have the $C^{1}$ continuity property embedded. We illustrate the first six of these, associated with a particular vertex $P_{1}$, in Fig. 2 . It is seen that unlike the Lagrange basis functions, these individual basis functions do not change sign within a triangle which might be an advantage in preserving positivity for physical quantities such as the density or pressure.

All of the integrals that need to be done to define the matrices that occur in the Galerkin method are of the form of 2D integrals of polynomials in $\xi$ and $\eta$ over the triangles. It is possible to convert these to sums of integrals that can each be done analytically by making use of the formula:

$$
F(m, n) \equiv \iint_{\text {triangle }} \xi^{m} \eta^{n} d \xi d \eta=c^{n+1} \frac{\left[a^{m+1}-(-b)^{m+1}\right] m ! n !}{(m+n+2) !}
$$

Thus, all integrations are done in closed form to machine precision.

Consider a common integral (traditionally called the mass matrix) over the triangle that occurs when we apply the Galerkin method to applications that will be discussed in the next section:

$$
\begin{aligned}
\iint v_{j}(\xi, \eta) \phi(\xi, \eta) d \xi d \eta & =\sum_{k=1}^{18}\left[\sum_{i=1}^{20} \sum_{l=1}^{20} g_{i, j} g_{l, k} F\left(m_{i}+m_{l}, n_{i}+n_{l}\right)\right] \Phi_{k} \\
& \equiv \sum_{k=1}^{18} M_{j, k} \Phi_{k}
\end{aligned}
$$


Other integrals needed for the applications presented, all of whose calculation is straightforward, are given in Appendix B.

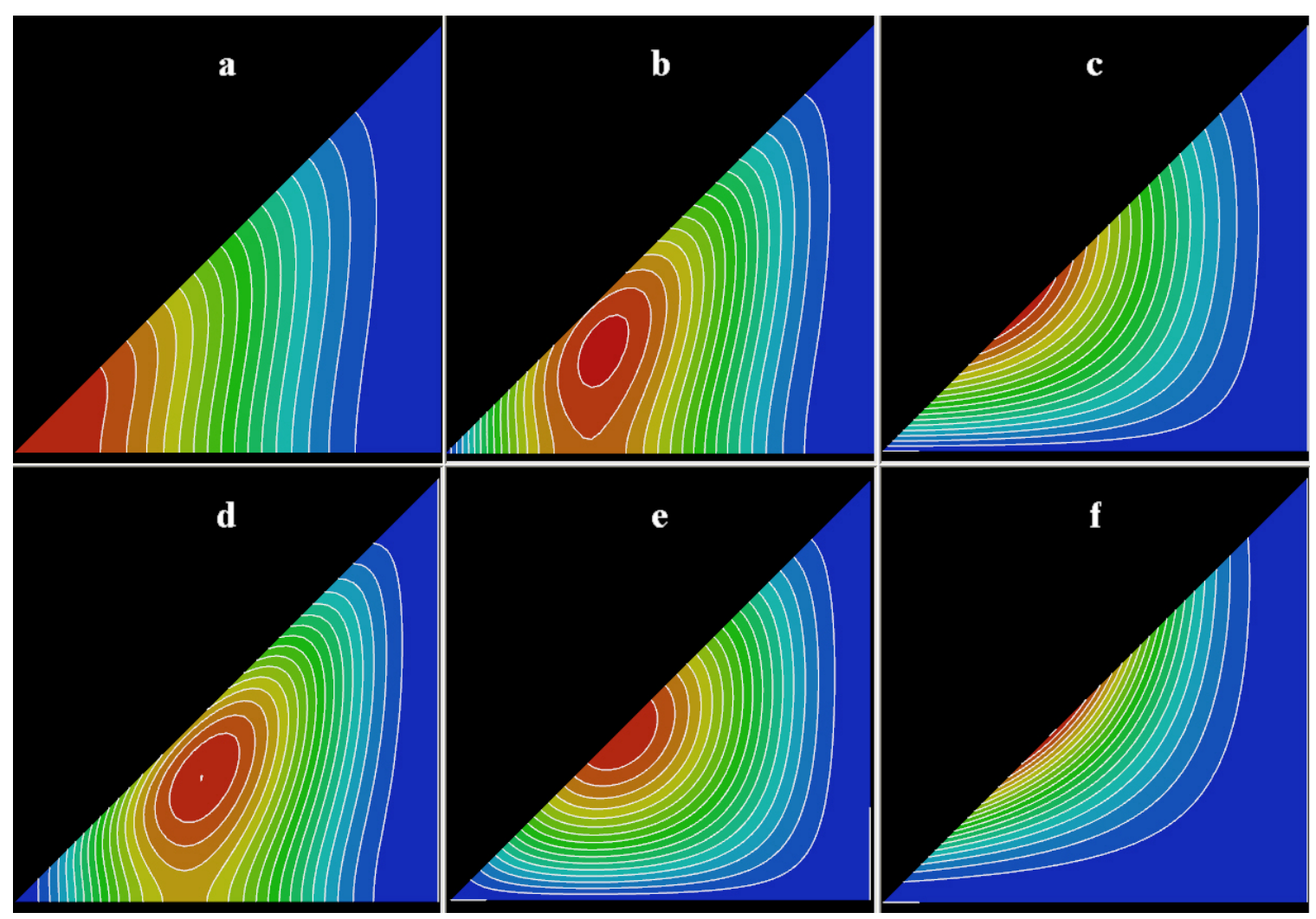

Figure 2: The 6 trial functions associated with the point $\mathbf{P}_{1}$ in the lower left corner. (a) $\phi=1$, (b) $\phi_{x}=1$, (c) $\phi_{y}=1$, (d) $\phi_{x x}=1$, (e) $\phi_{x y}=1$, (f) $\phi_{y y}=1$. None of the functions alternate sign. Red=1, Blue=0.

Essential boundary conditions are readily implemented by replacing the rows of the matrix $M_{i, j}$ in Eq. (1.6) corresponding to the function value or derivative for which a boundary condition is to be applied by a row with zeros everywhere except for the diagonal, in which there is placed a one. Then the boundary value of the corresponding function or derivative is placed in the corresponding location in the RHS vector.

Figure 3: Region is divided into regular rectangles, each of which is divided into two right triangles. Mesh (a) has 6 sides per vertex; Mesh (b) alternates 4 and 8 sides per vertex. (a)

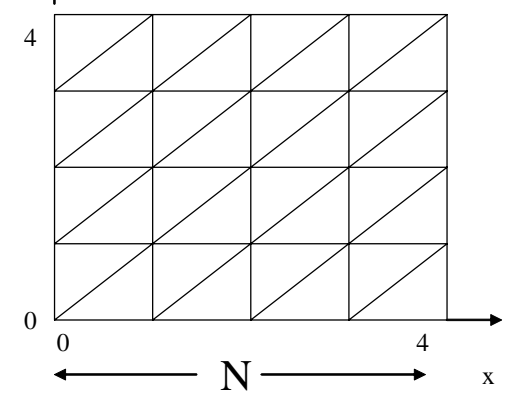

(b)

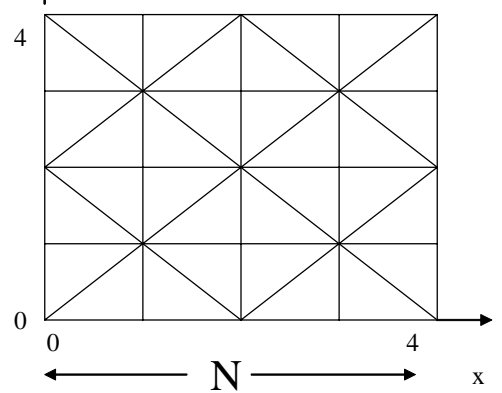




\section{Applications}

We present several examples that illustrate the accuracy and simplicity of this method. These applications are typical of those encountered in fusion MHD applications.

\section{a. A Simple Elliptic Problem}

Here we present a basic application of the method to a solution of Poisson's equation in a rectangular domain. Consider the equation:

$$
\nabla^{2} \Phi=f(x, y)
$$

We wish to solve Eq. (1.7) on the domain $0<x<L_{x} ; \quad 0<y<L_{y}$ with Dirichlet boundary conditions: $\Phi(0, y)=\Phi\left(L_{x}, y\right)=\Phi(x, 0)=\Phi\left(x, L_{x}\right)=0$. Equation (1.7) is equivalent to finding the function $\Phi(x, y)$ that minimizes the functional:

$$
I(\Phi)=\iint_{\substack{0<x<L_{x} \\ 0<y<L_{y}}}\left[\frac{1}{2}|\nabla \Phi|^{2}+f \Phi\right] d x d y
$$

For illustration, we choose the function $f(x, y)$ obtained by differentiating the exact solution: $\Phi(x, y)=x\left(x-L_{x}\right) y\left(y-L_{y}\right) \operatorname{sink} x$. For $L_{x}=L_{y}=4$, a square mesh is divided into $N^{2}$ regular square subdivisions, each of which is divided into two right triangles as shown in Fig. 3(a), so that there are a total of $2 N^{2}$ triangular elements with the linear dimension of each scaling like $1 / N$.

The integrals in Eq. (1.8) are evaluated in Appendix B. Minimization gives the matrix equation

$$
\mathbf{K \Phi}=\mathbf{F}
$$

which is solved for the unknown vector $\Phi$ using the sparse matrix direct solver routine SuperLU [11].

In Fig. 4 we plot the $L_{2}$ norm of the error in the solution for several values of $N$ and $k$, verifying that we obtain the expected $1 / N^{5}$ scaling. Note that there is approximately one wavelength per cell when $k=\pi N / 4$.

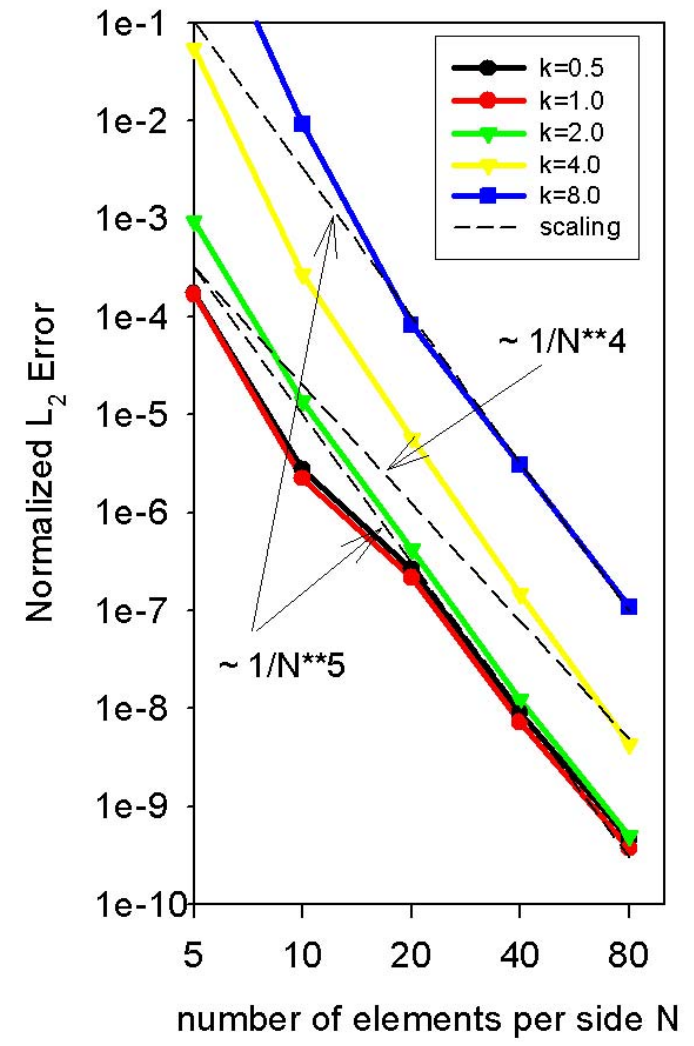

Figure 4: Elliptic equation exhibits $1 / \mathbf{N}^{5}$ scaling

\section{b. Anisotropic Thermal Conduction}

The second example is a demonstration of the accurate calculation of anisotropic thermal conduction. Let $\mathbf{B}$ be a 2-dimensional magnetic field written in terms of a given flux function $\psi(x, y)$, i.e., $\mathbf{B}=\hat{z} \times \nabla \psi$ and suppose the source function $S(x, y)$ is given, as are 
the two constants denoting the isotropic thermal conductivity $\kappa$, and the parallel thermal conductivity $\kappa_{\|}$. Consider the functional

$$
I=\iint\left[\frac{1}{2} \kappa_{\|}|\mathbf{B} \cdot \nabla \Phi|^{2}+\frac{1}{2} \kappa|\nabla \Phi|^{2}+S(x, y) \Phi\right]
$$

Minimizing this with respect to the unknown function $\Phi$ gives the steady state anisotropic heat conduction equation:

$$
\nabla \cdot \kappa_{\|} \mathbf{B B} \cdot \nabla \Phi+\nabla \cdot \kappa \nabla \Phi=S(x, y)
$$

The details of the evaluation of the matrix elements are given in Appendix B. For this application we let $\psi(x, y)=\sin \frac{\pi x}{L_{x}} \sin \frac{\pi y}{L_{y}}$, $\kappa=1$, and

$S(x, y)=\frac{\sin \left(\pi x / L_{x}\right) \sin \left(\pi y / L_{y}\right)}{\left(\pi / L_{x}\right)^{2}+\left(\pi / L_{y}\right)^{2}}$

Since the magnetic field flux function is proportional to the source function, it is readily verified that the solution should be independent of the value of the parallel conductivity $\kappa_{||}$, thus simplifying the error comparison.

The results are shown in Fig. 5. Again, we verify that at least $N^{5}$ scaling is obtained, and that reasonable accuracy $\left(10^{-5}\right)$ can be obtained for values of $\kappa_{\|} / \kappa$ as large as $10^{8}$ for values of $N$ as low as 60 . Note that the mesh

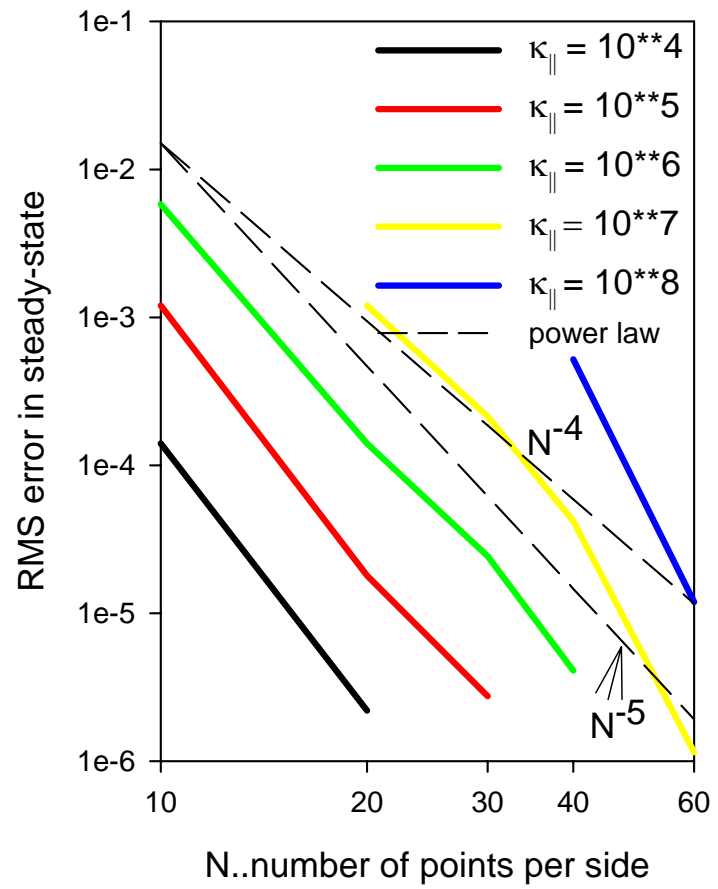

Figure 5: Convergence study shows $\mathbf{N}^{-5}$ convergence for anisotropic diffusion shown in Fig. 3(a) was again used, so that there was absolutely no attempt to align the element boundaries with the magnetic field direction for this demonstration.

\section{c. Ideal Tilting of an Incompressible Column}

The incompressible MHD equations in 2D can be written in terms of a stream function $\phi$ and a flux function $\psi$ using the normal Poisson bracket notation (where subscripts denote differentiation), i.e., $[f, g] \equiv f_{x} g_{y}-f_{y} g_{x}=f_{\xi} g_{\eta}-f_{\eta} g_{\xi}$

$$
\begin{aligned}
& \frac{\partial}{\partial t} \nabla^{2} \phi+\left[\nabla^{2} \phi, \phi\right]-\left[\nabla^{2} \psi, \psi\right]=\mu \nabla^{4} \phi \\
& \frac{\partial \psi}{\partial t}+[\psi, \phi]=\eta \nabla^{2} \psi
\end{aligned}
$$

Here $\mu$ and $\eta$ are constants denoting the plasma viscosity and resistivity, respectively. 
Note that these equations obey an energy theorem:

$$
\frac{\partial}{\partial t} \iint_{\text {domain }} \frac{1}{2}\left[|\nabla \phi|^{2}+|\nabla \psi|^{2}\right] d x d y=-\left[\eta\left|\nabla^{2} \psi\right|^{2}+\mu\left|\nabla^{2} \phi\right|^{2}\right]
$$

subject to the vanishing of $\partial \psi / \partial$, $\phi$, and $n \cdot \nabla \phi$ on the boundary.

Applying Galerkin's method to the set of equations (1.13), using the reduced quintic finite element, and applying $\theta$-weighted implicit finite differencing yields the following set of matrix equations to advance the solution from time $n$ to $n+1$ :

$$
\begin{aligned}
& {\left[\begin{array}{ll}
S_{j}^{11} & S_{j}^{12} \\
S_{j}^{21} & S_{j}^{22}
\end{array}\right]\left[\begin{array}{l}
\Phi_{j}^{n+1} \\
\Psi_{j}^{n+1}
\end{array}\right]=\left[\begin{array}{cc}
D_{j}^{11} & D_{j}^{12} \\
D_{j}^{21} & D_{j}^{22}
\end{array}\right]\left[\begin{array}{l}
\Phi_{j}^{n} \\
\Psi_{j}^{n}
\end{array}\right]} \\
& {\left[\begin{array}{ll}
S_{j}^{11} & S_{j}^{12} \\
S_{j}^{21} & S_{j}^{22}
\end{array}\right]=\left[\begin{array}{cc}
A_{i, j}+\theta \delta t\left[\bar{G}_{i, j, k} \Phi_{k}^{*}+\mu B_{i, j}\right] & -\theta \delta t \bar{G}_{i, j, k} \Psi_{k}^{*} \\
\theta \delta t K_{i, j, k} \Psi_{k}^{*} & M_{i, j}+\theta \delta t\left[K_{i, k, j} \Phi_{k}^{*}-\eta A_{i, j}\right]
\end{array}\right]} \\
& {\left[\begin{array}{cc}
D_{j}^{11} & D_{j}^{12} \\
D_{j}^{21} & D_{j}^{22}
\end{array}\right]=\left[\begin{array}{cc}
\left\{\begin{array}{c}
A_{i, j}-\delta t\left[G_{i, j, k} \Phi_{k}^{n}-\theta \bar{G}_{i, j, k} \Phi_{k}^{*}\right. \\
\left.+(1-\theta) \mu B_{i, j}\right]
\end{array}\right\} & \delta t\left(G_{i, j, k} \Psi_{k}^{n}-\theta \bar{G}_{i, j, k} \Psi_{k}^{*}\right) \\
\delta t K_{i, j, k}\left(-\frac{1}{2} \Psi_{k}^{n}+\theta \Psi_{k}^{*}\right)
\end{array} \quad\left\{\begin{array}{c}
M_{i, j}-\delta t\left[K_{i, k, j}\left(\frac{1}{2} \Phi_{k}^{n}-\theta \Phi_{k}^{*}\right)\right. \\
\left.-(1-\theta) \eta A_{i, j}\right]
\end{array}\right\}\right]}
\end{aligned}
$$

where $\bar{G}_{i, j, k} \equiv G_{i, j, k}+G_{i, k, j}$. The quantities occurring in the matrix are defined in the text and in Appendix B. Note that in the applications presented here, we set $\Phi^{*}=\Phi^{\mathrm{n}}$ and

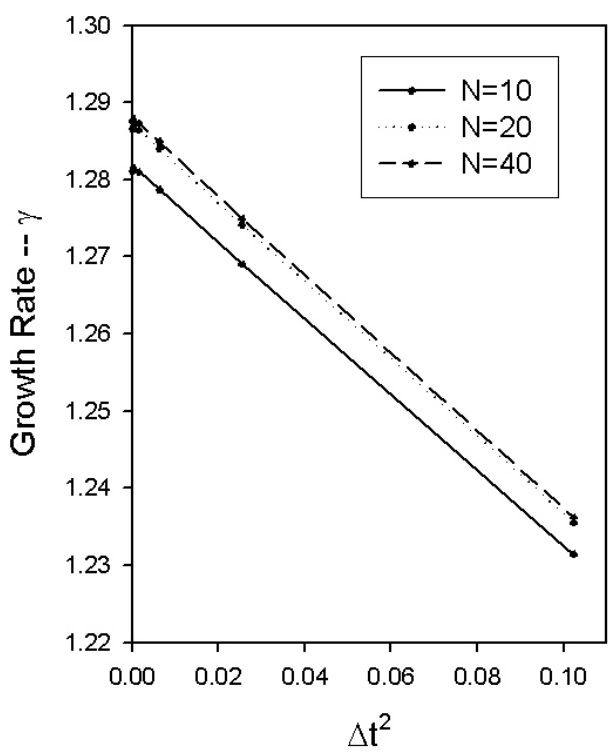

Figure 6: Convergence study of linear growth rate for tilt mode problem
$\Psi^{*}=\Psi^{\mathrm{n}}$, which necessitated inverting the matrix on the left in Eq. (1.15) each time step. However, it may be possible in many applications to obtain stable and accurate calculations by keeping $\Phi^{*}$ and $\Psi^{*}$ fixed for a number of time steps, thus significantly reducing the solution time. This is always the case in a linear calculation.

Following $[1,12]$ we define an initial bipolar vortex equilibrium state:

$$
\begin{aligned}
& \psi= \begin{cases}{\left[2 / k J_{0}(k)\right] J_{1}(k r) \cos \theta,} & r<1, \\
(r-1 / r) \cos \theta, & r>1,\end{cases} \\
& J_{1}(k)=0 .
\end{aligned}
$$

When perturbed, an instability occurs, growing exponentially as exp $\gamma$ t. The simulation box is again the square in Fig. 3(a) 
with sides of length 4 that is divided into $N \times N$ rectangular regions, each with 2 triangles. Conducting, no slip boundary conditions are applied at the wall: $\phi=0, n \cdot \nabla \phi=0, \partial \psi / \partial=0$. The first and second tangential derivatives of these quantities are also set to zero.

We show in Fig. 6 the dependence of the linear growth rate $\gamma$ on the size of the time step $\Delta t$ for a sequence of runs with $\mu=0.005, \eta=0.001, \theta=0.5$, and varying number of rectangular regions per dimension $\mathrm{N}$. For the smallest time step used, $\delta \mathrm{t}=0.01$, the growth rate $\gamma$ was 1.2876 , changing only in the $6^{\text {th }}$ decimal place when varying $\mathrm{N}$ from 30 to 40 , making a further convergence study in $\mathrm{N}$ unnecessary.

In the nonlinear stages, near singular current sheets form and the resolution requirements become more demanding. We plot the maximum perturbed current density vs. time (as determined from taking the maximum value on a $400 \times 400$ evaluation grid) for a run with $(\mu, \eta)=\left(5 . \times 10^{-3}, 10^{-4}\right)$ in Fig. 7 for three different linear resolutions, $N=20,30,40$. We monitor energy conservation as to how accurately Eq. (1.14) is satisfied. The calculation used an initial time step of $\delta \mathrm{t}=0.02$, which was reduced when the energy conservation was violated by more than $1 \%$. When $\delta \mathrm{t}<$ 0.0002 , the calculation was stopped.

Figure 7: Maximum perturbed current in the tilt-mode calculation as a function of time for three resolutions

We see from Fig. 7 that the calculations with $N=20,30,40$ give essentially the same results until the singularity begins its exponential growth, and that the $\mathrm{N}=40$ calculation can follow the singularity to about 4-times the height of the $\mathrm{N}=20$ calculation while still maintaining energy

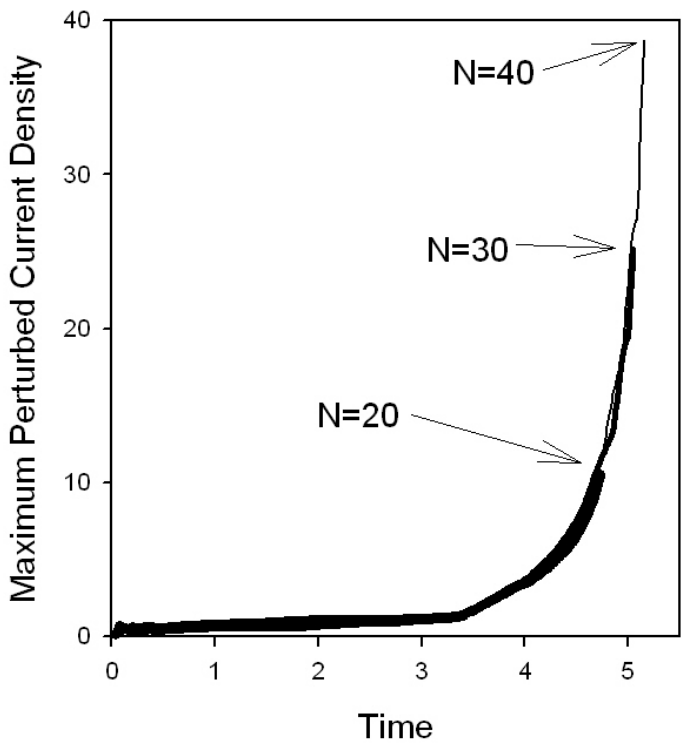
conservation to within $1 \%$. If we relax this stringent energy conservation requirement, the calculations would proceed much further without failing.

These calculations were repeated for $\mathrm{N}=40$ using the second mesh system shown in Fig. 3 as (b). We find essentially the same results, with the growth rate of the linear mode changing only beyond the $6^{\text {th }}$ decimal place, and the eigenfunctions appearing identical.

Appendix C presents a form of the above equations that is especially convenient for looking at small deviations from an equilibrium configuration (linearized displacements). The linearized application is particularly efficient for a direct solver (SuperLU) as it requires only a single $L U$ decomposition for a time dependent problem, and just a back substitution each time step. 
Appendix D combines the two equations into a higher order equation for the stream function $\phi$ that does not require the solution of the $\psi$ equation. This leads to a very efficient implicit time advance that highlights the advantages of using $C^{1}$ continuity elements.

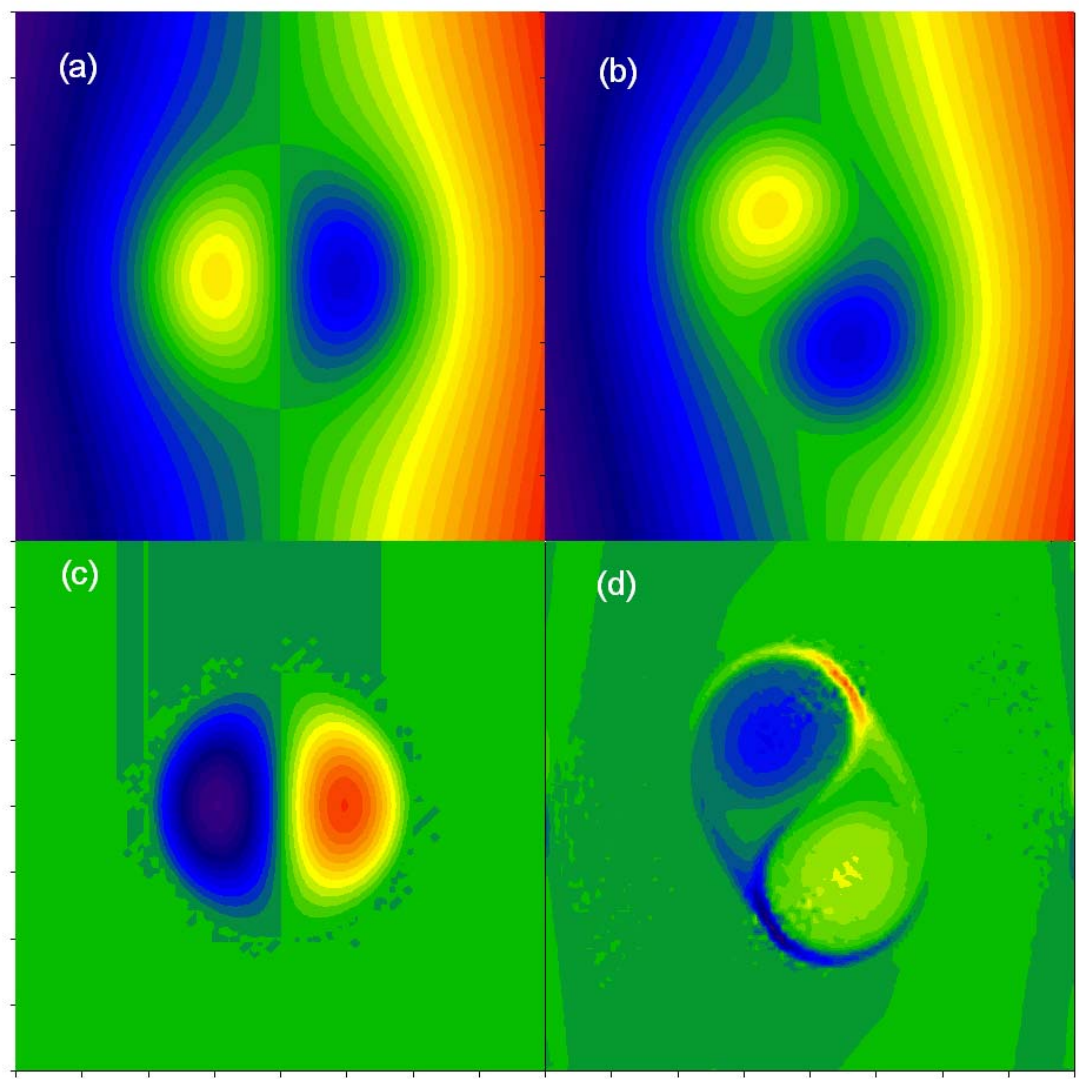

Figure 8: Poloidal flux at times $t=0$ (a) and $t=5$ (b) and plasma current at times $t=0$ (c) and $t=5$ (d) for the tilt mode problem with $\mathrm{N}=40$. The singular currents can be seen developing in (d)

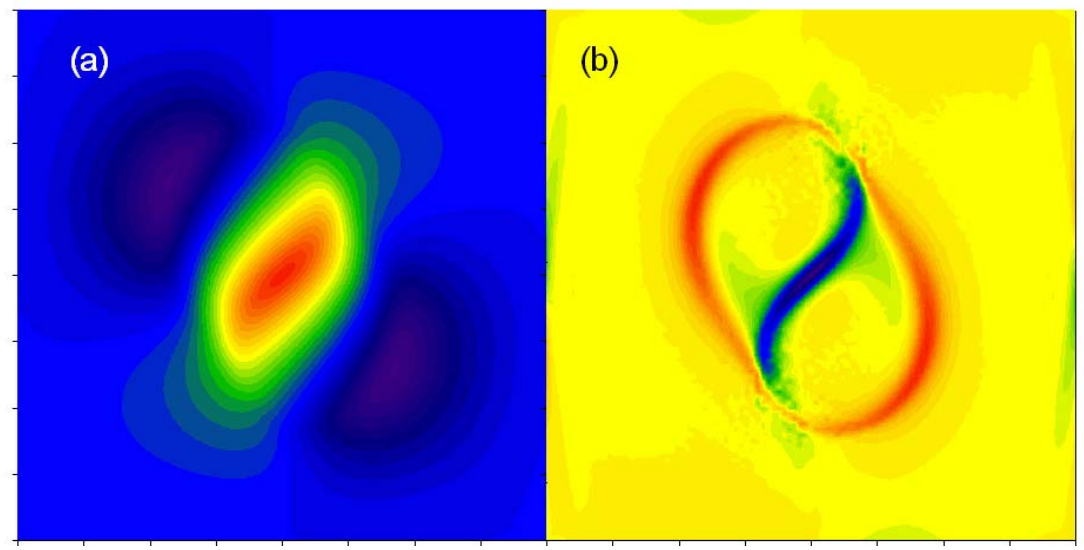

Figure 9: Stream function (a) and vorticity (b) for the tilt mode problem with $\mathrm{N}=40$ at time $t=5$. 


\section{d. The Grad-Shafranov-Schlüter (GSS) Equation}

The equation that the poloidal magnetic flux function satisfies in force-balance for a 2D axisymmetric plasma equilibrium is well known to be:

$$
\frac{\partial}{\partial x} \frac{1}{x} \frac{\partial \psi}{\partial x}+\frac{\partial}{\partial y} \frac{1}{x} \frac{\partial \psi}{\partial y}=-\left(x p^{\prime}(\psi)+\frac{1}{x} g g^{\prime}(\psi)\right)
$$

Here, $p(\psi)$ is the plasma pressure, $g(\psi)$ is the toroidal field function so that $g(\psi) / x$ is the toroidal field strength, and prime denotes a derivative with respect to $\psi$, the solution. To fully specify the problem, one must prescribe the two functions $p^{\prime}(\psi)$ and $g g^{\prime}(\psi)$ along with the boundary values for $\psi$. It is convenient to define the normalized flux function as $\tilde{\psi} \equiv\left(\psi-\psi_{0}\right) / \Delta \psi$, where we denote by $\psi_{0}$ the value of the poloidal flux at the magnetic axis, and by $\psi_{L}$ the value at the plasma-vacuum boundary, which is defined by the value of $\psi$ at a specified limiter location $\left(x_{L}, y_{L}\right)$. We further define the flux depth of the plasma as $\Delta \psi \equiv \psi_{L}-\psi_{0}$ so that values of $0 \leq \tilde{\psi}<1$ reside in the plasma, and values $1 \leq \tilde{\psi}$ are in the surrounding vacuum region. For these studies, we define the pressure and toroidal field functions as functions of the normalized poloidal flux function, $p=p(\tilde{\psi})$ and $g^{2}=g^{2}(\tilde{\psi})$, with the functional form specified as follows:

$$
\begin{array}{r}
p(s)=p_{0}\left[1+p_{1} s+p_{2} s^{2}-\left(20+10 p_{1}+4 p_{2}\right) s^{3}+\left(45+20 p_{1}+6 p_{2}\right) s^{4}\right. \\
\left.-\left(36+15 p_{1}+4 p_{2}\right) s^{5}+(10+4 p 1+p 2) s^{6}\right]
\end{array}
$$

and

$$
g^{2}(s)=g_{0}^{2}(s)+\gamma_{1} G_{1}(s)+\gamma_{2} G_{2}(s)+\gamma_{3} G_{3}(s)
$$

where

$$
\begin{aligned}
& G_{1}(s)=s-10 s^{3}+20 s^{4}-15 s^{5}+4 s^{6} \\
& G_{2}(s)=s^{2}-4 s^{3}+6 s^{4}-4 s^{5}+s^{6} \\
& G_{3}(s)=1-20 s^{3}+45 s^{4}-36 s^{5}+10 s^{6}
\end{aligned}
$$

These functional forms have been chosen so that the plasma current and pressure will go smoothly to zero at the plasma-vacuum boundary. The pressure function is then specified in terms of the three constants, $p_{0}, p_{1}$, and $p_{2}$. The three constants appearing in the toroidal field function, $\gamma_{1}, \gamma_{2}$, and $\gamma_{3}$, are used to prescribe the total plasma current $I_{p}$, the normalized reciprocal current density on axis $q_{0}$, and the slope of the current density near the axis $J_{\psi}$, respectively. The constant $g_{0}$ is the value of the toroidal field function due to the external fields. Thus, the constants appearing in Eq. (1.19) are given by:

$$
\begin{aligned}
& \gamma_{1}=-R_{0}\left(R_{0} p_{0} p_{1}+2 g_{0} \Delta \psi / R_{0}^{2} q_{0}\right) \\
& \gamma_{2}=-\left(J_{\psi} \Delta \psi / 2+p_{0} p_{2}\right) \\
& \gamma_{3}=-\left(I_{P}+\gamma_{1} I_{1}+\gamma_{2} I_{2}+I_{0}\right) / I_{3}
\end{aligned}
$$

The required integrals are obtained by first expressing the $x$ and $y$ derivatives of the functions $x p^{\prime}(\psi), \frac{1}{2 x} G_{1}^{\prime}(\psi), \frac{1}{2 x} G_{2}^{\prime}(\psi), \frac{1}{2 x} G_{3}^{\prime}(\psi)$ at each node in terms of the unknown 
vector $\boldsymbol{\Psi} \equiv\left[\psi, \psi_{x}, \psi_{y}, \psi_{x x}, \psi_{x y}, \psi_{y y}\right]$ and then using the finite element expansion (1.3) to extend these over the triangles so that the integrals can be performed in closed form. Thus,

$$
\begin{aligned}
& I_{0}=\iint_{\text {plasma }} x p^{\prime}(\psi) d x d y=\sum_{l=1}^{N} \sum_{j=1}^{18} C_{j}\left(x p^{\prime}\right)_{j} \\
& I_{k}=\iint_{\text {plasma }} \frac{1}{2 x} G_{k}^{\prime}(\psi) d x d y=\sum_{l=1}^{N} \sum_{j=1}^{18} C_{j}\left(\frac{1}{2 x} G_{k}^{\prime}\right)_{j} \quad, k=1,3
\end{aligned}
$$

where the first sum is over the $N$ triangular elements, and we have denoted by $\left(x p^{\prime}\right)_{j}$, etc, the value of the function in brackets and it's derivatives through second order with respect to $x$ and $y$ at each of the three nodes defining each triangle. The integrating factor appearing in Eq. (1.22) is given by $C_{j}=\sum_{p=1}^{20} g_{p, j} F\left(m_{p}, n_{p}\right)$. These integrals and constants are recomputed each iteration as $\psi$ changes.

The Galerkin method, together with a Picard iteration for the nonlinear equation (1.17) consists of multiplying by each test function, performing an integration by parts, integrating over the domain, and applying the iteration scheme:

$$
\mathbf{A} \cdot \Psi^{\mathbf{n + 1}}=\mathbf{B}\left(\boldsymbol{\Psi}^{\mathrm{n}}\right)
$$

where the matrix and vector elements are given in Appendix B. The boundary values are given using an analytic formula for the vector potential due to a filament source plus a uniform dipole field, which is required for equilibrium. Thus, at a boundary point of the domain $\left(x_{b}, y_{b}\right)$, the unknown $\psi$ and its tangential derivatives are calculated from the formula:

$$
\psi\left(x_{b}, y_{b}\right)=I_{P}\left[G\left(x_{b}, y_{b} ; x_{0}, y_{0}\right)+B_{V}\left(x_{b}^{2}-x_{0}^{2}\right) / 2\right]
$$

where

$$
\begin{aligned}
G\left(x_{b}, y_{b} ; x_{0}, y_{0}\right) & =\frac{\sqrt{x_{b} x_{0}}}{2 \pi k}\left[\left(2-k^{2}\right) K\left(k^{2}\right)-2 E\left(k^{2}\right)\right] \\
k^{2} & =\frac{4 x_{b} x_{0}}{\left(x_{b}+x_{0}\right)^{2}+\left(y_{b}-y_{0}\right)^{2}} \\
B_{V} & =\frac{1}{4 \pi x_{0}}\left[\ln \left(\frac{8 x_{0}}{a}\right)-\frac{3}{2}+\left(\frac{\ell_{i}}{2}+\beta_{P}\right)\right]
\end{aligned}
$$

with $K\left(k^{2}\right)$ and $E\left(k^{2}\right)$ being the complete elliptic integrals of the first and second kind, $a$ is the plasma minor radius, defined by $a^{2}=\left(x_{0}-x_{L}\right)^{2}+\left(z_{0}-z_{L}\right)^{2}$, and $\ell_{i}$ and $\beta_{P}$ are the plasma internal inductance and poloidal beta. These are enforced by zeroing out the corresponding row of $\mathbf{A}$ in Eq. (1.23), and inserting a one on the diagonal, and the boundary value in the appropriate location in $\mathbf{B}$. This is done for $\psi$ and its first two tangential derivatives. 
In the results presented here, we computed on the rectangular domain: $10<\mathrm{x}<14,-2<\mathrm{y}$ $<2$, that was divided into $2 N^{2}$ equally spaced triangular elements. Other parameters were $\left(x_{0}, y_{0}\right)=(12.1,0),.\left(x_{L}, y_{L}\right)=(10.5,0),. p_{0}=0.01, p_{1}=-1 ., p_{2}=0 ., I_{P}=1 ., g_{0}=36.4, q_{0}=$ 1., $J_{\psi}=0$, and we set $\left(\ell_{\mathrm{i}} / 2+\beta_{\mathrm{P}}\right)=1.2$.

This gives a value of $\psi_{0}=-6.165228$, changing only in the $7^{\text {th }}$ decimal place for $N \geq 15$. We plot in Fig. 10 the $L_{2}$ error in Eq. (1.17) as a function of $N$. This is defined by directly evaluating each side of Eq. (1.17) at each node point in the plasma region, squaring the difference, summing these, and taking the square root of the sum divided by the number of node points summed. It is seen that the error converges approximately as $\mathrm{N}^{-3.5}$. We postulate that this behavior is due to the fact that the functions in Eq. (1.17) only have continuous derivatives through second order at the plasma-vacuum interface, and thus the higher order terms in the expansion are not completely effective in reducing the error further.

\section{Summary and Discussion}

We have shown that the reduced quintic 2D triangular finite element is well-suited for many problems arising in fusion MHD applications. It is easy to work with, and has excellent convergence properties if the actual solution is smooth enough.

We have demonstrated it's applicability on a 2D elliptic problem, in the solution of the anisotropic heat conduction problem, in a time-dependent reduced-MHD problem and for the $2 \mathrm{D}$ axisymmetric toroidal equilibrium problem.

The element requires only three unknowns per triangle, which is considerably less than other highorder elements of comparable accuracy (Table 2). The fact that it forces $C^{1}$ continuity, and is thus

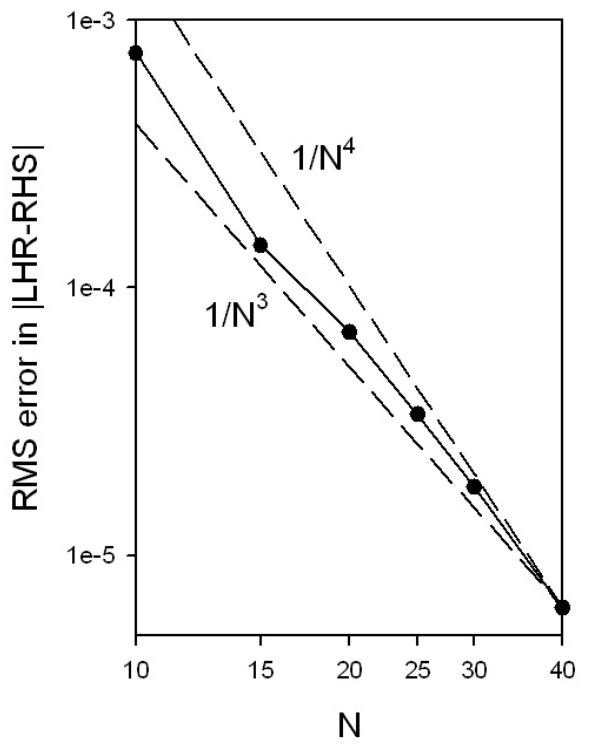

Figure 10: RMS error in GSS equation as a function of elements per side. Convergence is $\sim \mathbf{N}^{-3.5}$ suitable for problems involving derivatives up to fourth order in space makes it very efficient for systems of equations that can be combined into a smaller number of higher order equations.

This property to handle higher order equations was utilized in Appendix D to cast the reduced incompressible resistive MHD equations in a fully implicit form that consisted of two sequential sparse matrix linear solves, each of rank $(3 N)^{2}$ for a problem with $N$ triangles. We can contrast this to $C^{0}$ methods of comparable accuracy, which would have to solve the combined system together (more variables), and would also have more unknowns per triangle per variable, resulting in considerably larger matrices.

It has been recognized since the 1970s that the reduced quintic finite element has many advantageous properties. In [6] it is referred to as "one of the most interesting and 
ingenious of all finite elements" and it states that "a series of careful numerical experiments has given first prize to this remarkable element", referring to the studies in reference [10]. The example problems presented in this paper support the notion that this element offers many advantages for extended MHD calculations. Future studies will focus on a more complete system of equations, and on the application of the element to irregular domains.

\section{Acknowledgements}

This work was supported by US DoE contract DE-AC02-76CH03073. The author has benefited from discussions with his colleagues, in particular P. Fischer, A. Glasser, W. Park, G. Richter, R. Samtaney, C. Sovinec, and H. Strauss. S. Klasky has assisted in the preparation of the figures.

\section{References}

1. $\quad$ H. R. Strauss and D. W. Longcope, J. Comput. Phys, 147, 318-336 (1998)

2. W. Park, E. V. Belova, G. Y. Fu, et al, Phys Plasmas 6 1796-1803 Part 2 May 1999

3. C. R. Sovinec, A. H. Glasser, G. A. Gianakon, et al, "Nonlinear magnetohydrodynamics simulations using high-order finite elements”, J. Comput Phys. To appear 2004

4. $\quad$ P. Fischer, "Spectral Element Solution of Anisotropic Diffusion in a Tokamak Model Geometry", in press

5. Glasser, A. H., "The SEL macroscopic modeling code”, Computer Physics Communications, to appear 2004

6. G. Strang and G. Fix, "An Analysis of the Finite Element Method”, Prentice-Hall Inc., 1973

7. D. Braess, "Finite Elements”, Cambridge University Press, 2002

8. K. Bell, "Anaylsis of Thin Plates in Bending Using Triangular Finite Elements", Feb. 1968, Institutt for Statikk, Norges Tekniske Hogskole, Tronkheim

9. J.H. Argyris, J. Fried, and D. W. Sharpf, "The TUBA Family of Plate Elements for the Matrix Displacement Method”, The Aeronautical Journal, 72, 1968 pp. 701-709 10. G. R. Cowper, E. Kosko, G. M. Lindberg, and M. D. Olson, AIAA Journal, 7 1957 (1969)

11. J.W. Demmel, J.R. Gilbert, Y. S. Li, “SuperLU Users Guide”, U.C. Berkeley, October 2003.

12. R. Richard, R. D. Sydora, and M. Ashour-abdalla, Phys. Fluids B 2, 488 (1990) 


\section{Appendix A: The transformation matrix}

To derive the transformation matrix $g_{i, j}$, we first calculate the value of $\phi$ and it's derivatives with respect to the local Cartesian coordinates $\xi$ and $\eta$ at the three vertex points, and combine this with the two constraint equations enforcing $C^{1}$ continuity along the edges. (Note that the third constraint was automatically satisfied in removing the $21^{\text {st }}$ coefficient in the sum). Using the expansion $\phi(\xi, \eta)=\sum_{i=1}^{20} a_{i} \xi^{m_{i}} \eta^{n_{i}}$, the two additional constraint equations become:

$5 b^{4} c a_{16}+\left(3 b^{2} c^{3}-2 b^{4} c\right) a_{17}+\left(2 b c^{4}-3 b^{3} c^{2}\right) a_{18}+\left(c^{5}-4 b^{2} c^{3}\right) a_{19}-5 b c^{4} a_{20}=0$

$5 a^{4} c a_{16}+\left(3 a^{2} c^{3}-2 a^{4} c\right) a_{17}+\left(-2 a c^{4}-3 a^{3} c^{2}\right) a_{18}+\left(c^{5}-4 a^{2} c^{3}\right) a_{19}-5 a c^{4} a_{20}=0$

and thus the transformation matrix $\mathbf{T}$ in the local coordinates takes the form:

$=\left[\begin{array}{cccccccccccccccccccc}1 & -b & 0 & b^{2} & 0 & 0 & -b^{3} & 0 & 0 & 0 & b^{4} & 0 & 0 & 0 & 0 & -b^{5} & 0 & 0 & 0 & 0 \\ 0 & 1 & 0 & -2 b & 0 & 0 & 3 b^{2} & 0 & 0 & 0 & -4 b^{3} & 0 & 0 & 0 & 0 & 5 b^{4} & 0 & 0 & 0 & 0 \\ 0 & 0 & 1 & 0 & -b & 0 & 0 & b^{2} & 0 & 0 & 0 & -b^{3} & 0 & 0 & 0 & 0 & 0 & 0 & 0 & 0 \\ 0 & 0 & 0 & 2 & 0 & 0 & -6 b & 0 & 0 & 0 & 12 b^{2} & 0 & 0 & 0 & 0 & -20 b^{3} & 0 & 0 & 0 & 0 \\ 0 & 0 & 0 & 0 & 1 & 0 & 0 & -2 b & 0 & 0 & 0 & 3 b^{2} & 0 & 0 & 0 & 0 & 0 & 0 & 0 & 0 \\ 0 & 0 & 0 & 0 & 0 & 2 & 0 & 0 & -2 b & 0 & 0 & 0 & 2 b^{2} & 0 & 0 & 0 & -2 b^{3} & 0 & 0 & 0 \\ 1 & a & 0 & a^{2} & 0 & 0 & a^{3} & 0 & 0 & 0 & a^{4} & 0 & 0 & 0 & 0 & a^{5} & 0 & 0 & 0 & 0 \\ 0 & 1 & 0 & 2 a & 0 & 0 & 3 a^{2} & 0 & 0 & 0 & 4 a^{3} & 0 & 0 & 0 & 0 & 5 a^{4} & 0 & 0 & 0 & 0 \\ 0 & 0 & 1 & 0 & a & 0 & 0 & a^{2} & 0 & 0 & 0 & a^{3} & 0 & 0 & 0 & 0 & 0 & 0 & 0 & 0 \\ 0 & 0 & 0 & 2 & 0 & 0 & 6 a & 0 & 0 & 0 & 12 a^{2} & 0 & 0 & 0 & 0 & 20 a^{3} & 0 & 0 & 0 & 0 \\ 0 & 0 & 0 & 0 & 1 & 0 & 0 & 2 a & 0 & 0 & 0 & 3 a^{2} & 0 & 0 & 0 & 0 & 0 & 0 & 0 & 0 \\ 0 & 0 & 0 & 0 & 0 & 2 & 0 & 0 & 2 a & 0 & 0 & 0 & 2 a^{2} & 0 & 0 & 0 & 2 a^{3} & 0 & 0 & 0 \\ 1 & 0 & c & 0 & 0 & c^{2} & 0 & 0 & 0 & c^{3} & 0 & 0 & 0 & 0 & c^{4} & 0 & 0 & 0 & 0 & c^{5} \\ 0 & 1 & 0 & 0 & c & 0 & 0 & 0 & c^{2} & 0 & 0 & 0 & 0 & c^{3} & 0 & 0 & 0 & 0 & c^{4} & 0 \\ 0 & 0 & 1 & 0 & 0 & 2 c & 0 & 0 & 0 & 3 c^{2} & 0 & 0 & 0 & 0 & 4 c^{3} & 0 & 0 & 0 & 0 & 5 c^{4} \\ 0 & 0 & 0 & 2 & 0 & 0 & 0 & 2 c & 0 & 0 & 0 & 0 & 2 c^{2} & 0 & 0 & 0 & 0 & 2 c^{3} & 0 & 0 \\ 0 & 0 & 0 & 0 & 1 & 0 & 0 & 0 & 2 c & 0 & 0 & 0 & 0 & 3 c^{2} & 0 & 0 & 0 & 0 & 4 c^{3} & 0 \\ 0 & 0 & 0 & 0 & 0 & 2 & 0 & 0 & 0 & 6 c & 0 & 0 & 0 & 0 & 12 c^{2} & 0 & 0 & 0 & 0 & 20 c^{3} \\ 0 & 0 & 0 & 0 & 0 & 0 & 0 & 0 & 0 & 0 & 0 & 0 & 0 & 0 & 0 & 5 a^{4} c & 3 a^{2} c^{3}- & -2 a c^{4}+ & c^{5}- & 5 a c^{4} \\ 0 & 0 & 0 & 0 & 0 & 0 & 0 & 0 & 0 & 0 & 0 & 0 & 0 & 0 & 0 & 5 b^{4} c & 3 b^{2} c^{3}- & 2 b c^{3}- & 4 a^{5} c^{3} & 5 b^{4} c^{2} \\ 3 & & & & & & & & & & & & & & & & 3 b^{3} c^{2} & 4 b^{2} c^{3} & -5 b c^{4}\end{array}\right]$

This satisfies $\boldsymbol{\Phi}^{\prime}=\mathbf{T} \mathbf{A}$, where $\boldsymbol{\Phi}^{\prime}$ denotes the vector produced by stringing together the function and derivatives with respect to $\xi$ and $\eta$ [Cartesian coordinates that are rotated with respect to $(x, y)$.] at the three vertices, and with the final two elements zero: i.e. $\boldsymbol{\Phi}^{\prime}=\left[\phi^{1}, \phi_{\xi}^{1}, \phi_{\eta}^{1}, \phi_{\xi \xi}^{1}, \phi_{\xi \eta}^{1}, \phi_{\eta \eta}^{1}, \phi^{2}, \ldots, \phi^{3}, \ldots, 0,0\right]$ This can be solved for the coefficient matrix by inverting $\mathbf{T}$, thus $\mathbf{A}=\mathbf{T}^{-1} \boldsymbol{\Phi}^{\prime}$. A useful check is to verify that the numerically evaluated determinant of $\mathbf{T}$ has the value $-64(a+b)^{17} c^{20}\left(a^{2}+c^{2}\right)\left(b^{2}+c^{2}\right)$. Note that since the final two elements of $\boldsymbol{\Phi}^{\prime}$ are zero, we can replace $\mathbf{T}^{-1}$ by the $20 \times 18$ matrix $\mathbf{T}_{2}$ which consists of the first 18 columns of $\mathbf{T}^{-1}$. 
To get the coefficient matrix $\mathbf{A}$ in terms of the vector containing the actual derivatives with respect to $(x, y)$, we have to apply the rotation matrix $\mathbf{R}$. This is compactly defined in terms of the angle $\theta$ appearing in Fig. 1 by:

$$
\mathbf{R}=\left[\begin{array}{lll}
\mathbf{R}_{1} & & \\
& \mathbf{R}_{1} & \\
& & \mathbf{R}_{1}
\end{array}\right]
$$

where

$$
\mathbf{R}_{\mathbf{1}}=\left[\begin{array}{cccccc}
1 & 0 & 0 & 0 & 0 & 0 \\
0 & \cos \theta & \sin \theta & 0 & 0 & 0 \\
0 & -\sin \theta & \cos \theta & 0 & 0 & 0 \\
0 & 0 & 0 & \cos ^{2} \theta & 2 \sin \theta \cos \theta & \sin ^{2} \theta \\
0 & 0 & 0 & -\sin \theta \cos \theta & \cos ^{2} \theta-\sin ^{2} \theta & \sin \theta \cos \theta \\
0 & 0 & 0 & \sin ^{2} \theta & -2 \sin \theta \cos \theta & \cos ^{2} \theta
\end{array}\right]
$$

Thus, if we define the matrix $\mathbf{G}=\mathbf{T}_{\mathbf{2}} \mathbf{R}$, this relates the coefficient matrix directly to the unknown vector consisting of the function and derivatives with respect to $(x, y)$, thus: $\mathbf{A}=\mathbf{G} \boldsymbol{\Phi}$, or in component notation: $a_{i}=\sum_{j=1}^{18} g_{i, j} \Phi_{j}$ for $i=1,20$. 


\section{Appendix B: Matrix Elements}

The most basic matrix element was given in Eq. (1.6). Here we give the remaining ones that occur in the example problems that have been presented. In obtaining these results, we perform integration by parts as required to equalize the number of derivatives operating on the test and trial functions.

$$
\begin{gathered}
\iint v_{j}(\xi, \eta) \nabla^{2} \phi(\xi, \eta) d \xi d \eta=\sum_{k=1}^{18} A_{j, k} \Phi_{k} \\
A_{j, k}=\sum_{p=1}^{20} \sum_{q=1}^{20} g_{p, j} g_{q, k}\left[m_{p} m_{q} F\left(m_{p}+m_{q}-2, n_{p}+n_{q}\right)+n_{p} n_{q} F\left(m_{p}+m_{q}, n_{p}+n_{q}-2\right)\right] \\
\iint v_{j}(\xi, \eta) \nabla^{4} \phi(\xi, \eta) d \xi d \eta=\sum_{k=1}^{18} B_{j, k} \Phi_{k} \\
B_{j, k}=-\sum_{p=1}^{20} \sum_{q=1}^{20} g_{p, j} g_{q, k}\left\{\begin{array}{l}
m_{p}\left(m_{p}-1\right) m_{q}\left(m_{q}-1\right) \times F\left(m_{p}+m_{q}-4, n_{p}+n_{q}\right) \\
+n_{p}\left(n_{p}-1\right) n_{q}\left(n_{q}-1\right) \times F\left(m_{p}+m_{q}, n_{p}+n_{q}-4\right) \\
+\left[m_{p}\left(m_{p}-1\right) n_{q}\left(n_{q}-1\right)+m_{q}\left(m_{q}-1\right) n_{p}\left(n_{p}-1\right)\right] \\
\times F\left(m_{p}+m_{q}-2, n_{p}+n_{q}-2\right)
\end{array}\right\}
\end{gathered}
$$

In $2 \mathrm{D}$, if the magnetic field is written as $\mathbf{B}=\hat{z} \times \nabla \psi$, and if $\psi$ has the expansion as in Eq. (1.3), i.e., $\psi=\sum_{i=1}^{18} v_{i} \Psi_{i}$, then we can compute the matrix element:

$$
\begin{gathered}
\iint v_{j}(\xi, \eta) \nabla \cdot \mathbf{B B} \cdot \nabla \Phi d \xi d \eta=\iint\left[v_{j}, \psi\right][\phi, \psi] d \xi d \eta \\
=\sum_{k=1}^{18} R_{j, k} \Phi_{k} \\
R_{j, k} \equiv \sum_{i=1}^{18} \sum_{l=1}^{18} \sum_{p=1}^{20} \sum_{q=1}^{20} \sum_{r=1}^{20} \sum_{s=1}^{20} g_{p, j} g_{q, i} g_{r, k} g_{s, l}\left(m_{p} n_{q}-m_{q} n_{p}\right)\left(m_{r} n_{s}-m_{s} n_{r}\right) \times \\
F\left(m_{p}+m_{q}+m_{r}+m_{s}-2, n_{p}+n_{q}+n_{r}+n_{s}-2\right) \Psi_{i} \Psi_{l} \\
\iint_{i}(\xi, \eta)\left[\nabla^{2} \psi, \psi\right] d \xi d \eta=\sum_{j=1}^{18} \sum_{k=1}^{18} G_{i, j, k} \Psi_{j} \Psi_{k} \\
G_{i, j, k}=\sum_{p=1}^{20} \sum_{q=1}^{20} \sum_{r=1}^{20} g_{p, i} g_{q, j} g_{r, k}\left(m_{p} n_{r}-m_{r} n_{p}\right)\left[\begin{array}{l}
m_{q}\left(m_{q}-1\right) F\left(m_{p}+m_{q}+m_{r}-3, n_{p}+n_{q}+n_{r}-1\right) \\
\left.+n_{q}\left(n_{q}-1\right) F\left(m_{p}+m_{q}+m_{r}-1, n_{p}+n_{q}+n_{r}-3\right)\right]
\end{array}\right.
\end{gathered}
$$




$$
\begin{aligned}
& \iint v_{i}(\xi, \eta)[\psi, \phi] d \xi d \eta=\sum_{j=1}^{18} \sum_{k=1}^{18} K_{i, j, k} \Psi_{j} \Phi_{k} \\
& K_{i, j, k}=\sum_{p=1}^{20} \sum_{q=1}^{20} \sum_{r=1}^{20} g_{p, i} g_{q, j} g_{r, k}\left(m_{q} n_{r}-m_{r} n_{q}\right) F\left(m_{p}+m_{q}+m_{r}-1, n_{p}+n_{q}+n_{r}-1\right)
\end{aligned}
$$

Suppose $f(x, z)$ is a function with a known Taylor's series expansion about the origin of each triangle:

$$
\begin{aligned}
f(x, z) & =\sum_{k=0}^{4} \sum_{l=0}^{k} \frac{1}{l !(k-l) !}\left[\frac{\partial^{k} f}{\partial x^{l} \partial z^{k-l}}\right]_{x_{0}, z_{0}}\left(x-x_{0}\right)^{l}\left(z-z_{0}\right)^{k-l} \\
& =\sum_{k=0}^{4} \sum_{l=0}^{k}\left[\frac{\partial^{k} f}{\partial x^{l} \partial z^{k-l}}\right]_{x_{0}, z_{0}} \sum_{p=0}^{l} \frac{(\cos \theta)^{l-p}(-\sin \theta)^{p}}{p !(l-p) !} \sum_{q=0}^{k-l} \frac{(\sin \theta)^{k-l-q}(\cos \theta)^{q}}{q !(k-l-q) !} \xi^{k-p-q} \eta^{p+q}
\end{aligned}
$$

Then, we can compute:

$$
\begin{aligned}
& \iint\left[v_{j}^{i} f\right] d x d z=\sum_{k=0}^{4} \sum_{l=0}^{k} \sum_{p=0}^{l} \sum_{q=0}^{k-l} M_{k l p q}^{i} F\left(k-p-q+m_{j}, p+q+n_{j}\right) \\
& M_{k l p q}^{i}=\left[\frac{\partial^{k} f}{\partial x^{l} \partial z^{k-l}}\right]_{x_{0}, z_{0}} \frac{\left(\cos \theta_{i}\right)^{l-p}\left(-\sin \theta_{i}\right)^{p}}{p !\left(\operatorname{lin} \theta_{i}\right)^{k-l-q}\left(\cos \theta_{i}\right)^{q}} \\
& q !(k-l-q) !
\end{aligned}
$$

where the index $i$ refers to the number of the triangle.

In order to evaluate the differential operator appearing in the GSS equation, we first expand the function $(1 / x)$ in terms of it's derivatives as in Eq. (1.3), i.e. $1 / x=\sum_{k=1}^{18} v_{k}(1 / x)_{k}$. Using this, we can calculate the matrix element

$$
\begin{aligned}
& \iint_{\text {element }} v_{j}\left(\frac{\partial}{\partial x} \frac{1}{x} \frac{\partial \psi}{\partial x}+\frac{\partial}{\partial y} \frac{1}{x} \frac{\partial \psi}{\partial y}\right) d x d y=\sum_{k=1}^{18} I_{k, j} \Psi_{j} \\
& I_{k, j}=\sum_{i=1}^{18} \sum_{p=1}^{20} \sum_{q=1}^{20} \sum_{r=1}^{20} g_{p, i} g_{q, k} g_{r, j}\left[\begin{array}{l}
m_{q} m_{r} F\left(m_{p}+m_{q}+m_{r}-2, n_{p}+n_{q}+n_{r}\right) \\
+n_{q} n_{r} F\left(m_{p}+m_{q}+m_{r}, n_{p}+n_{q}+n_{r}-2\right)
\end{array}\right]\left(\frac{1}{x}\right)_{i}
\end{aligned}
$$

The terms appearing on the right of Eq. (1.17) are readily calculated once terms like $x p^{\prime}(\psi)$ are expanded in terms of their derivatives, i.e. $x p^{\prime}=\sum_{j=1}^{18} v_{j}\left(x p^{\prime}\right)_{j}$, thus

$$
\iint_{\text {triangle }} v_{j} x p^{\prime} d x d y=\sum_{k=1}^{18} \sum_{p=1}^{20} \sum_{q=1}^{20} g_{p, j} g_{q, k} F\left(m_{p}+m_{q}, n_{p}+n_{q}\right)\left(x p^{\prime}\right)_{k}
$$




\section{Appendix C: Alternate form for incompressible MHD matrices:}

The matrices in Eq. (1.15) can also be written in terms of the deviation of the solution from an initial equilibrium. Thus, if we define the vector:

$$
\left[\begin{array}{c}
\tilde{\Phi}_{j}^{n} \\
\tilde{\Psi}_{j}^{n}
\end{array}\right] \equiv\left[\begin{array}{c}
\Phi_{j}^{n} \\
\Psi_{j}^{n}
\end{array}\right]-\left[\begin{array}{c}
\Phi_{j}^{0} \\
\Psi_{j}^{0}
\end{array}\right]
$$

The matrix equations can be written

$$
\begin{gathered}
{\left[\begin{array}{ll}
S_{j}^{\prime 11} & S_{j}^{\prime 2} \\
S_{j}^{\prime 21} & S_{j}^{\prime 22}
\end{array}\right]\left[\begin{array}{c}
\tilde{\Phi}_{j}^{n+1} \\
\tilde{\Psi}_{j}^{n+1}
\end{array}\right]=\left[\begin{array}{cc}
D_{j}^{\prime 11} & D_{j}^{\prime 2} \\
D_{j}^{\prime 21} & D_{j}^{\prime 22}
\end{array}\right]\left[\begin{array}{c}
\tilde{\Phi}_{j}^{n} \\
\tilde{\Psi}_{j}^{n}
\end{array}\right]} \\
{\left[\begin{array}{ll}
S_{j}^{\prime 11} & S_{j}^{\prime 2} \\
S_{j}^{\prime 21} & S_{j}^{\prime 22}
\end{array}\right]=\left[\begin{array}{cc}
A_{i, j}+\theta \delta t\left[\bar{G}_{i, j, k}\left(\tilde{\Phi}_{k}^{*}+\Phi_{k}^{0}\right)+\mu B_{i, j}\right] & -\theta \delta t \bar{G}_{i, j, k}\left(\tilde{\Psi}_{k}^{*}+\Psi_{k}^{0}\right) \\
\theta \delta t K_{i, j, k}\left(\tilde{\Psi}_{k}^{*}+\Psi_{k}^{0}\right) & M_{i, j}+\theta \delta t\left[K_{i, k, j}\left(\tilde{\Phi}_{k}^{*}+\Phi_{k}^{0}\right)-\eta A_{i, j}\right]
\end{array}\right]} \\
{\left[\begin{array}{cc}
D_{j}^{\prime 11} & D_{j}^{\prime 12} \\
D_{j}^{\prime 21} & D_{j}^{\prime 22}
\end{array}\right]=\left[\begin{array}{cc}
A_{i, j}-\delta t \bar{G}_{i, j, k}\left(\frac{1}{2} \tilde{\Phi}_{k}^{n}+\Phi_{k}^{0}\right. & \delta t \bar{G}_{i, j, k}\left[\frac{1}{2} \tilde{\Psi}_{k}^{n}+\Psi_{k}^{0}\right. \\
\left.-\theta\left(\tilde{\Phi}_{k}^{*}+\Phi_{k}^{0}\right)+(1-\theta) \mu B_{i, j}\right] & \left.-\theta\left(\tilde{\Psi}_{k}^{*}+\Psi_{k}^{0}\right)\right] \\
-\delta t K_{i, j, k}\left[\frac{1}{2} \tilde{\Psi}_{k}^{n}+\Psi_{k}^{0}\right. & M_{i, j}-\delta t\left\{K_{i, j, k}\left[\frac{1}{2} \tilde{\Phi}_{k}^{n}+\Phi_{k}^{0}-\theta\left(\tilde{\Phi}_{k}^{*}+\Phi_{k}^{0}\right)\right]\right. \\
\left.-\theta\left(\tilde{\Psi}_{k}^{*}+\Psi_{k}^{0}\right)\right] & \left.-(1-\theta) \eta A_{i, j}\right\}
\end{array}\right]}
\end{gathered}
$$

This form allows the matrices to be evaluated only once per problem for a linear calculation. In this case, the LU decomposition is performed only once at the outset, and Eq. (1.37) is solved every timestep with a matrix multiplication and the LU backsubstitution. 


\section{Appendix D: A second alternative form for the reduced MHD equations matrices.}

We note that it is possible to eliminate $\dot{\psi}$ from the time advancement equation for $\phi$ in Eq. (1.13). After applying the $\theta$-centered time differencing, this yields the set of time advance equations:

$$
\begin{gathered}
\left\{\nabla^{2}+\theta \delta t L_{1}+(\theta \delta t)^{2} L_{2}\right\} \tilde{\Phi}^{n+1}= \\
-\theta \delta t^{2} L_{2} \Phi^{0}+\delta t R \\
S^{\prime 22} \tilde{\Psi}^{n+1}=-S^{\prime 21} \tilde{\Phi}^{n+1}+D^{\prime 21} \tilde{\Phi}^{n}+D^{\prime 22} \tilde{\Psi}^{n}
\end{gathered}
$$

The feature of this formulation is that Eq. (1.39) does not involve $\tilde{\Psi}^{n+1}$, and so these two equations can be solved in series, resulting in a much faster solution time compared to the formulation given in Eq. (1.15).

We have defined the operators

$$
\begin{aligned}
L_{1} \tilde{\Phi}^{n+1}= & {\left[\nabla^{2} \tilde{\Phi}^{n+1}, \tilde{\Phi}\right]+\left[\nabla^{2} \tilde{\Phi}^{n+1}, \Phi^{0}\right]+\left[\nabla^{2} \tilde{\Phi}, \tilde{\Phi}^{n+1}\right]+\left[\nabla^{2} \Phi^{0}, \tilde{\Phi}^{n+1}\right]-\mu \nabla^{4} \tilde{\Phi}^{n+1} } \\
L_{2} \tilde{\Phi}^{n+1}= & {\left[\nabla^{2} \tilde{\Psi}+\nabla^{2} \Psi^{0}\left[\tilde{\Psi}+\Psi^{0}, \tilde{\Phi}^{n+1}\right]\right]-\left[\left[\tilde{\Phi}^{n+1}, \nabla^{2} \tilde{\Psi}+\nabla^{2} \Psi^{0}\right], \tilde{\Psi}+\Psi^{0}\right] } \\
& -\left[\left[\nabla^{2} \tilde{\Phi}^{n+1}, \tilde{\Psi}+\Psi^{0}\right], \tilde{\Psi}+\Psi^{0}\right]-2\left[\left[\tilde{\Phi}_{x}^{n+1}, \tilde{\Psi}_{x}+\Psi_{x}^{0}\right], \tilde{\Psi}+\Psi^{0}\right] \\
& -2\left[\left[\tilde{\Phi}_{y}^{n+1}, \tilde{\Psi}{ }_{y}+\Psi_{y}^{0}\right], \tilde{\Psi}+\Psi^{0}\right] \\
R= & -\left[\nabla^{2} \tilde{\Phi}^{n}, \tilde{\Phi}\right]-\left[\nabla^{2} \Phi^{0}, \tilde{\Phi}^{n}\right]-\left[\nabla^{2} \tilde{\Phi}^{n}, \Phi^{0}\right] \\
& +\left[\nabla^{2} \tilde{\Psi}^{n}, \tilde{\Psi}^{n}\right]+\left[\nabla^{2} \Psi^{0}, \tilde{\Psi}^{n}\right]+\left[\nabla^{2} \tilde{\Psi}^{n}, \Psi^{0}\right]+\mu \nabla^{4} \tilde{\Phi}
\end{aligned}
$$

Evaluation of the terms in Eq. (1.39) requires computation of the new integrals that appear in the matrix elements. For any functions $\phi, \psi, \zeta$ with corresponding vectors $\Psi, \Phi, \mathrm{Z}$ :

$$
\begin{aligned}
\iint v_{i}(\xi, \eta)\left[\left[\phi, \nabla^{2} \psi\right], \zeta\right] d \xi d \eta & =\iint \nabla^{2} \psi\left[\phi,\left[v_{i}, \zeta\right]\right] d \xi d \eta \\
& =\sum_{j=1}^{18} \sum_{k=1}^{18} \sum_{l=1}^{18} P_{i, j, k, l} \Psi_{j} \Phi_{k} \mathrm{Z}_{l}
\end{aligned}
$$

where

$$
P_{i, j, k, l}=\sum_{p=1}^{20} \sum_{q=1}^{20} \sum_{r=1}^{20} \sum_{s=1}^{20} g_{p, i} g_{q, j} g_{r, k} g_{s, l}\left[\begin{array}{l}
{\left[m_{r}\left(n_{p}+n_{s}-1\right)-n_{r}\left(m_{p}+m_{s}-1\right)\right]\left(m_{p} n_{s}-m_{s} n_{p}\right) \times} \\
\left\{\begin{array}{l}
m_{q}\left(m_{q}-1\right) F\left(m_{p}+m_{q}+m_{r}+m_{s}-4, n_{p}+n_{q}+n_{r}+n_{s}-2\right) \\
+n_{q}\left(n_{q}-1\right) F\left(m_{p}+m_{q}+m_{r}+m_{s}-2, n_{p}+n_{q}+n_{r}+n_{s}-4\right)
\end{array}\right\}
\end{array}\right]
$$


similarly,

$$
\begin{aligned}
\iint v_{i}(\xi, \eta)\left[\nabla^{2} \psi,[\zeta, \phi]\right] d \xi d \eta & =\iint \nabla^{2} \psi\left[v_{i},[\phi, \zeta]\right] d \xi d \eta \\
& =\sum_{j=1}^{18} \sum_{k=1}^{18} \sum_{l=1}^{18} P_{k, j, i, l} \Psi_{j} \Phi_{k} \mathrm{Z}_{l},
\end{aligned}
$$

Other needed relations follow from the permutation symmetry of the Poisson bracket, thus

$$
P_{i, j, k, l}=-P_{l, j, k, i}
$$

We further define

$$
\begin{aligned}
\iint v_{i}(\xi, \eta)\left\{\left[\left[\phi_{x}, \psi_{x}\right], \zeta\right]+\left[\left[\phi_{y}, \psi_{y}\right], \zeta\right]\right\} d \xi d \eta & =\iint\left\{\left[\psi_{x}, \phi_{x}\right]+\left[\psi_{y}, \phi_{y}\right]\right\}\left[v_{i}, \zeta\right] d \xi d \eta \\
& =\sum_{j=1}^{18} \sum_{k=1}^{18} \sum_{l=1}^{18} R_{i, j, k, l} \Phi_{j} \Psi_{k} Z_{l}
\end{aligned}
$$

where

$$
\left.R_{i, j, k, l}=\sum_{p=1}^{20} \sum_{q=1}^{20} \sum_{r=1}^{20} \sum_{s=1}^{20} g_{p, i} g_{q, j} g_{r, k} g_{s, l}\left[\begin{array}{l}
\left(m_{p} n_{s}-m_{s} n_{p}\right) \times \\
m_{q} m_{r}\left[\left(m_{r}-1\right) n_{q}-\left(m_{q}-1\right) n_{r}\right] \times \\
F\left(m_{p}+m_{q}+m_{r}+m_{s}-4, n_{p}+n_{q}+n_{r}+n_{s}-2\right) \\
+n_{q} n_{r}\left[m_{r}\left(n_{q}-1\right)-m_{q}\left(n_{r}-1\right)\right] \times \\
F\left(m_{p}+m_{q}+m_{r}+m_{s}-2, n_{p}+n_{q}+n_{r}+n_{s}-4\right)
\end{array}\right\}\right)
$$

Multiplying Eq. (1.39) by each test function, integrating over the triangles, and using these and previous definitions, we obtain:

$$
\begin{gathered}
\iint v_{i} \nabla^{2} \tilde{\Phi} d \xi d \eta=\sum_{j=1}^{18} A_{i, j} \tilde{\Phi}_{j} \\
\iint v_{i} L_{1}\{\tilde{\Phi}\} d \xi d \eta=\sum_{j=1}^{18}\left[-\mu B_{i, j}+\sum_{k=1}^{18}\left(G_{i, j, k}+G_{i, k, j}\right)\left(\tilde{\Phi}_{k}+\Phi_{k}^{0}\right)\right] \tilde{\Phi}_{j} \\
\iint v_{i} L_{2}\{\tilde{\Phi}\} d \xi d \eta=\sum_{j=1}^{18} \sum_{k=1}^{18} \sum_{l=1}^{18}\left[P_{j, k, i, l}+P_{j, l, i, k}+\left(\begin{array}{l}
P_{i, j, k, l}+P_{i, j, l, k}-P_{i, k, j, l}-P_{i, l, j, k} \\
-2 R_{i, j, k, l}-2 R_{i, j, l, k}
\end{array}\right)\right] \times \\
\frac{1}{2}\left(\Psi_{l}^{0}+\tilde{\Psi}_{l}\right)\left(\Psi_{k}^{0}+\tilde{\Psi}_{k}\right) \tilde{\Phi}_{j}
\end{gathered}
$$

and finally

$$
\iint v_{i} R d \xi d \eta=\sum_{j=1}^{18}\left[\mu B_{i, j} \tilde{\Phi}_{j}^{n}+\sum_{k=1}^{18}\left(G_{i, j, k}+G_{i, k, j}\right)\left[\left(\frac{1}{2} \tilde{\Psi}_{k}^{n}+\Psi_{k}^{0}\right) \tilde{\Psi}_{j}^{n}-\left(\frac{1}{2} \tilde{\Phi}_{k}^{n}+\Phi_{k}^{0}\right) \tilde{\Phi}_{j}^{n}\right]\right]
$$




\section{External Distribution}

Plasma Research Laboratory, Australian National University, Australia

Professor I.R. Jones, Flinders University, Australia

Professor João Canalle, Instituto de Fisica DEQ/IF - UERJ, Brazil

Mr. Gerson O. Ludwig, Instituto Nacional de Pesquisas, Brazil

Dr. P.H. Sakanaka, Instituto Fisica, Brazil

The Librarian, Culham Laboratory, England

Mrs. S.A. Hutchinson, JET Library, England

Professor M.N. Bussac, Ecole Polytechnique, France

Librarian, Max-Planck-Institut für Plasmaphysik, Germany

Jolan Moldvai, Reports Library, Hungarian Academy of Sciences, Central Research Institute for Physics, Hungary

Dr. P. Kaw, Institute for Plasma Research, India

Ms. P.J. Pathak, Librarian, Institute for Plasma Research, India

Ms. Clelia De Palo, Associazione EURATOM-ENEA, Italy

Dr. G. Grosso, Instituto di Fisica del Plasma, Italy

Librarian, Naka Fusion Research Establishment, JAERI, Japan

Library, Laboratory for Complex Energy Processes, Institute for Advanced Study, Kyoto University, Japan

Research Information Center, National Institute for Fusion Science, Japan

Dr. O. Mitarai, Kyushu Tokai University, Japan

Dr. Jiangang Li, Institute of Plasma Physics, Chinese Academy of Sciences, People's Republic of China

Professor Yuping Huo, School of Physical Science and Technology, People's Republic of China

Library, Academia Sinica, Institute of Plasma Physics, People's Republic of China

Librarian, Institute of Physics, Chinese Academy of Sciences, People's Republic of China

Dr. S. Mirnov, TRINITI, Troitsk, Russian Federation, Russia

Dr. V.S. Strelkov, Kurchatov Institute, Russian Federation, Russia

Professor Peter Lukac, Katedra Fyziky Plazmy MFF UK, Mlynska dolina F-2, Komenskeho Univerzita, SK-842 15 Bratislava, Slovakia

Dr. G.S. Lee, Korea Basic Science Institute, South Korea

Institute for Plasma Research, University of Maryland, USA

Librarian, Fusion Energy Division, Oak Ridge National Laboratory, USA

Librarian, Institute of Fusion Studies, University of Texas, USA

Librarian, Magnetic Fusion Program, Lawrence Livermore National Laboratory, USA

Library, General Atomics, USA

Plasma Physics Group, Fusion Energy Research Program, University of California at San Diego, USA

Plasma Physics Library, Columbia University, USA

Alkesh Punjabi, Center for Fusion Research and Training, Hampton University, USA

Dr. W.M. Stacey, Fusion Research Center, Georgia Institute of Technology, USA

Dr. John Willis, U.S. Department of Energy, Office of Fusion Energy Sciences, USA

Mr. Paul H. Wright, Indianapolis, Indiana, USA 
The Princeton Plasma Physics Laboratory is operated by Princeton University under contract with the U.S. Department of Energy.

\author{
Information Services \\ Princeton Plasma Physics Laboratory \\ P.O. Box 451 \\ Princeton, NJ 08543
}

Phone: 609-243-2750

Fax: 609-243-2751

e-mail: pppl_info@pppl.gov

Internet Address: http://www.pppl.gov 